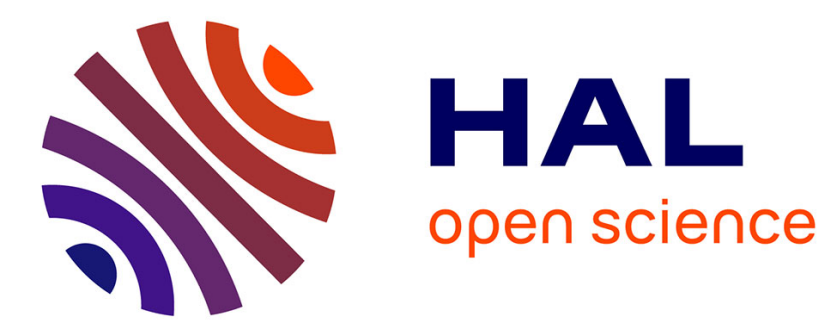

\title{
ON THE VOLUME OF A LINE BUNDLE
}

Sébastien Boucksom

\section{To cite this version:}

Sébastien Boucksom. ON THE VOLUME OF A LINE BUNDLE. International Journal of Mathematics, 2002, 13 (10), pp.1043-1063. 10.1142/S0129167X02001575 . hal-02105200

\section{HAL Id: hal-02105200 \\ https://hal.science/hal-02105200}

Submitted on 20 Apr 2019

HAL is a multi-disciplinary open access archive for the deposit and dissemination of scientific research documents, whether they are published or not. The documents may come from teaching and research institutions in France or abroad, or from public or private research centers.
L'archive ouverte pluridisciplinaire HAL, est destinée au dépôt et à la diffusion de documents scientifiques de niveau recherche, publiés ou non, émanant des établissements d'enseignement et de recherche français ou étrangers, des laboratoires publics ou privés. 


\title{
On the volume of a line bundle
}

\author{
Sébastien Boucksom
}

February 1, 2008

\begin{abstract}
Author's address: Institut Fourier, 100 rue des Maths, BP74, 38402 Saint-Martin d'Hères Cedex, France.

$e-$ mail: sbouckso@ujf-grenoble.fr

Abstract: Using a result of Fujita on approximate Zariski decompositions and the singular version of Demailly's holomorphic Morse inequalities as obtained by Bonavero, we express the volume of a line bundle in terms of the absolutely continuous parts of all the positive curvature currents on it, with a way to pick an element among them which is most homogeneous with respect to the volume. This enables us to introduce the volume of any pseudoeffective class on a compact Kähler manifold, and Fujita's theorem is then extended to this context.
\end{abstract}

2000 Mathematics Subject Classification: 32J25, 32J27, 14C20

\section{Introduction}

For a holomorphic line bundle $L$ on a compact complex manifold $X$ of dimension $n$, one defines the volume of $L$ as

$$
v(L):=\limsup _{k \rightarrow+\infty} \frac{n !}{k^{n}} h^{0}(X, k L) .
$$

Then $L$ is big, i.e. it has maximal Kodaira-Iitaka dimension $\kappa(X, L)=n$, exactly when $v(L)>0$. If $L$ is an ample line bundle, the combination of Serre's vanishing theorem and the asymptotic Riemann-Roch formula shows that $v(L)=L^{n}$, where $L^{n}$ is the $n$-fold intersection number $\int_{X} c_{1}(L)^{n}$. When $L$ is merely a nef (numerically effective) line bundle and $X$ is Kähler, one can show using Demailly's Morse inequalities that $h^{q}(X, k L)=o\left(k^{n}\right)$ for $q>0$, so that the Riemann-Roch formula again yields $v(L)=L^{n}$ in that case.

Recall now that a line bundle $L$ is said to have an algebraic Zariski decomposition if there exists two Q-line bundles $N$ and $E$, which are nef and effective respectively, such that 
(i) $L=N+E$ as Q-line bundles.

(ii) $H^{0}(X, k L)=H^{0}(X, k N)$ for every positive integer $k$ clearing up the denominator of $N$.

Then one of course has $v(L)=v(N)=N^{n}$, so that the knowledge of the nef part $N$ enables one to compute the volume of $L$. On a surface, such a decomposition exists, but this is no longer true in general, even if one allows $X$ to be blown up; however the following result, due to T.Fujita ([Fu94], cf. also [DEL00]), shows that in some sense it is still true asymptotically.

Theorem 1.1 (Approximate Zariski decomposition) Let L be a big line bundle on the projective manifold $X$. Then for every $\varepsilon$ there exists a finite sequence of blowing-ups with smooth centers $\mu: \widetilde{X} \rightarrow X$ and two Q-line bundles $A$ and $E$ on $\widetilde{X}$ which are ample and effective respectively (the data depends on $\varepsilon)$ such that:

(i) $L=A+E$ as $\mathbf{Q}$-line bundles.

(ii) $v(A) \leq v(L) \leq v(A)+\varepsilon$

One would like to say that in general an algebraic Zariski decomposition of $L$ could be obtained by letting $\varepsilon$ tend to zero in the above theorem, which cannot be done in an algebraic context. Our first endeavour is to translate the theorem of Fujita into the language of singular Hermitian metrics on $L$ (here the main tool is the Calabi-Yau theorem), so as to get in the limit a "most homogeneous" metric on $L$ with respect to the volume. More precisely, we prove the following:

Theorem 1.2 Let $L$ be a pseudoeffective line bundle on a compact Kähler manifold $X$. Then:

(i) $v(L)=\sup _{T} \int_{X} T_{a c}^{n}$ for $T$ ranging among the closed positive $(1,1)$-currents in the cohomology class $c_{1}(L)$.

(ii) Given a Kähler form $\omega$ on $X$, normalized so that $\int_{X} \omega^{n}=1$, there exists a closed positive $(1,1)$-current $T \in c_{1}(L)$ such that $T_{a c}^{n}(x)=v(L) \omega^{n}(x)$ for almost every $x \in X$.

Recall that a line bundle $L$ is said to be pseudoeffective if it can be equipped with a singular Hermitian metric $h$ with positive curvature current $\Theta_{h}(L)$, or equivalently if the (de Rham) cohomology class $c_{1}(L)$ contains a closed positive $(1,1)$-current $T . T_{a c}$ is then the absolutely continuous part of $T$ in its Lebesgue decomposition $T=T_{a c}+T_{s g} . T_{a c}$ is considered as a $(1,1)$-form with $L_{l o c}^{1}$ coefficients, so that the product $T_{a c}^{n}$ is meant pointwise.

In particular, Theorem 1.2 shows that the volume $v(L)$ of $L$ only depends on the first Chern class $c_{1}(L)$. It therefore seems natural to introduce the following 
Definition 1.3 Let a be a pseudoeffective $(1,1)$-cohomology class on $X$ compact Kähler. Then one defines its volume by

$$
v(a):=\sup _{T} \int_{X} T_{a c}^{n}
$$

for $T$ ranging over the closed positive $(1,1)$-currents in a.

We show that the volume $v(a)$ is always finite, that it is continuous on the whole pseudoeffective cone and that Theorem 1.2 still holds in this more general context. Furthermore, we give the corresponding version of Fujita's theorem:

Theorem 1.4 Let $X$ be a compact Kähler manifold, and let a be a pseudoeffective $(1,1)$-cohomology class on $X$ with $v(a)>0$. Then for every $\varepsilon>0$ there exists a finite sequence of blowing-ups with smooth centers $\mu: \widetilde{X} \rightarrow X$, a Kähler class $\omega$ and an effective real divisor $D$ on $\widetilde{X}$ such that

(i) $\mu^{\star} a=\omega+D$ as cohomology classes.

(ii) $v(\omega)=\omega^{n} \leq v(a) \leq v(\omega)+\varepsilon$

In particular, a pseudoeffective class with positive volume is big, in the sense that it contains a Kähler current (cf. definitions below).

\section{Preliminary tools.}

\subsection{Currents with analytic singularities}

In this section, $X$ denotes a complex $n$-dimensional manifold. Recall first that

(i) A closed real $(1,1)$-current $T$ on $X$ is said to be almost positive if some continuous real $(1,1)$-form $\gamma$ can be found such that $T \geq \gamma$, and that a function $\varphi$ in $L_{l o c}^{1}(X)$ is almost plurisubharmonic (psh for short) if its Hessian $d d^{c} \varphi$ is an almost positive current (here $d d^{c}=\frac{i}{\pi} \partial \bar{\partial}$ ). This latter property is equivalent to the fact that $\varphi$ can locally be written as a sum of a plurisubharmonic function and a smooth one.

(ii) A closed (1,1)-current $T$ is called a Kähler current if one has $T \geq \omega$ for some Hermitian form $\omega$ on $X$. For example, a line bundle $L$ on $X$ complex compact is big if and only if it admits a curvature current which is Kähler. That the condition is sufficient is a consequence of a result of Bonavero [Bon93] which will be stated below (cf. also [JS93], or [Dem96] for the easier case where $X$ is Kähler). In the other direction, choose $k$ such that $H^{0}(X, k L)$ 
generates 1-jets generically, and fix some smooth metric $h_{\infty}$ on $L$. Then the metric $h:=h_{\infty} e^{-2 \varphi}$ has Kähler curvature current if one takes $\varphi:=\frac{1}{2 k} \log \left(\sum\left|\sigma_{j}\right|_{h_{\infty}}^{2}\right)$ for some basis $\left(\sigma_{j}\right)$ of $H^{0}(X, k L)$. But the fact that this metric is obtained algebraically has a nice consequence on the singularities of $h$. In fact, it has algebraic singularities in the following sense:

Definition 2.1 (i) Given a coherent ideal sheaf $\mathcal{I}$ and $c>0$, we say that a function $\varphi$ has singularities of type $(\mathcal{I}, c)$ if it can locally be written as $\varphi=\frac{c}{2} \log \left(\sum\left|f_{j}\right|^{2}\right)+\theta$ for some local generators $\left(f_{j}\right)$ of $\mathcal{I}$ and some smooth function $\theta$.

(ii) We say that $\varphi$ has analytic (resp. algebraic) singularities if it has singularities of type $(\mathcal{I}, c)$ for some ideal sheaf $\mathcal{I}$ and some real (resp. rational) $c>0$.

A function with analytic singularities is automatically almost psh, and we can use the same terminology about the singularities of a closed almost positive current by looking at its local potentials. One can translate algebraic data into potential theoretic ones by means of the

Proposition 2.2 Given $c>0$ and a coherent ideal sheaf $\mathcal{I}$, there exists an almost psh function $\varphi$ on $X$ with singularities of type $(\mathcal{I}, c)$.

The proof consists merely in gluing together by means of a partition of unity functions of the type $\frac{c}{2} \log \left(\sum_{j}\left|g_{j}\right|^{2}\right)$, where the $g_{j}$ are local generators of $\mathcal{I}$. To do this, cover $X$ by a finite number of open sets $U_{k}$ such that $\mathcal{I}$ is generated by $f_{k, j}, j=1, \ldots, N_{k}$ on $U_{k}$. Then take $\theta_{k}$ smooth, positive, with support in $U_{k}$, and such that $\sum_{k} \theta_{k}^{2}=1$. Then one sets $\varphi=\frac{c}{2} \log \left(\sum_{k} \theta_{k}^{2} e^{\varphi_{k}}\right)$ with $\varphi_{k}=\log \left(\sum_{j}\left|f_{k, j}\right|^{2}\right)$.

Let us stress that the data $(\mathcal{I}, c)$ is not uniquely determined for a function $\varphi$ with analytic singularities (for instance $\mathcal{I}$ can always be replaced by its integral closure). We have the following obvious functorial property of functions with analytic singularities:

Proposition 2.3 Let $f: X \rightarrow Y$ be a holomorphic map between complex manifolds, and let $\varphi$ have singularities of type $(\mathcal{I}, c)$. Then $f^{\star} \varphi$ has singularities of type $\left(f^{-1} \mathcal{I}, c\right)$.

Corollary 2.4 (Resolution of singularities) Given $\varphi$ with singularities described by $(\mathcal{I}, c)$ on $X$ compact complex, there exists a finite sequence of blowing-ups $\mu: \widetilde{X} \rightarrow X$ such that $\mu^{\star} \varphi$ has singularities along a divisor of $\widetilde{X}$ with normal crossings. 
This follows by first blowing up $X$ along $\mathcal{I}$ and then applying Hironaka's resolution of singularities.

Now recall Siu's decomposition formula: given a closed almost positive current $T$ of bidimension $(p, p)$, one can write

$$
T=\sum_{j} \nu_{j}\left[A_{j}\right]+R
$$

where the $A_{j}$ are irreducible $p$-dimensional analytic subsets,

$\nu_{j}=\min _{x \in A_{j}} \nu(T, x)$ is the generic Lelong number of $T$ along $A_{j}$, and $R$ is a closed almost positive $(1,1)$-current such that $\operatorname{dim} E_{c}(R)<p$ for every $c>0$ $\left(E_{c}(R)=\{x \in X, \nu(R, x) \geq c\}\right.$ is the Lelong sublevel set, which is analytic by a well known result of Y.T.Siu.) Then we claim the following

Proposition 2.5 For a closed (1,1)-current with singularities of type $(\mathcal{I}, c)$, the Siu decomposition coincides with the Lebesgue decomposition, i.e. $T_{a c}=R$ and $T_{s g}=\sum_{j} \nu_{j}\left[A_{j}\right]$. Furthermore, $T_{s g}$ is a finite sum which corresponds to the divisorial part of $V(\mathcal{I})$.

Indeed, working locally, suppose that $\varphi=\frac{1}{2} \log \left(\left|f_{1}\right|^{2}+\ldots+\left|f_{N}\right|^{2}\right)$, and let $g$ be the g.c.d. of the $f_{j}$ 's. Then $d d^{c} \varphi=D+R$ where $D=d d^{c} \log |g|$ is the integration current along the divisor $\{g=0\}$ (by the Poincaré-Lelong formula) and $R=\frac{1}{2} d d^{c} \log \left(\left|g_{1}\right|^{2}+\ldots+\left|g_{N}\right|^{2}\right)$, thus it suffices to show that $R$ is absolutely continuous. But it is even smooth outside the set $A$ of common zeroes of the $g_{j}$ 's, and carries no mass on $A$ since codim $A \geq 2$ by construction, thus it has $L_{l o c}^{1}$ coefficients.

Note that as a consequence $T_{a c}$ and $T_{s g}$ are closed for $T$ with analytic singularities, which is not true in general.

Now in general one has the following result due to Demailly [Dem92]:

Theorem 2.6 (Approximation with algebraic singularities) Let $T$ be a closed almost positive $(1,1)$-current on $X$ compact complex equipped with some Hermitian form $\omega$. Write $T=\alpha+d d^{c} \varphi$ for $\alpha$ smooth and $\varphi$ almost psh, and suppose also given some continuous real form $\gamma$ such that $T \geq \gamma$. Then there exists a sequence $\varphi_{k}$ of almost psh functions such that:

(i) $\varphi_{k}$ has algebraic singularities.

(ii) $\varphi_{k}$ decreases to $\varphi$ everywhere.

(iii) The Lelong numbers $\nu\left(\varphi_{k}, x\right)$ converge to $\nu(\varphi, x)$ uniformly with respect to $x$.

(iv) Setting $T_{k}=\alpha+d d^{c} \varphi_{k}$, one has $T_{k} \geq \gamma-\varepsilon_{k} \omega$ for some sequence $\varepsilon_{k}$ decreasing to 0 . 
For our concerns, we need a slight improvement of this result, in order to take care of the absolutely continuous parts in the convergence.

Corollary 2.7 In the above proposition, we can also impose (v) $T_{k, a c} \rightarrow T_{a c}$ almost everywhere.

Proof: We appeal to a different approximation result [Dem82] taking care of the absolutely continuous parts (it is essentially a convolution): there exists a sequence $\psi_{j}$ of smooth functions on $X$ such that, setting $S_{j}=\alpha+d d^{c} \psi_{j}$, we have:

(a) $\psi_{j}$ decreases to $\varphi$ everywhere.

(b) $S_{j} \geq \gamma-C \lambda_{j} \omega$ for some $C>0$ and continuous functions $\lambda_{j}$ decreasing pointwise to the Lelong number,

(c) $S_{j}(x) \rightarrow T_{a c}(x)$ almost everywhere.

What we will do is glue the two sequences $\varphi_{k}$ and $\psi_{j}$ in the following fashion: denote by $A_{k}$ the polar set of $\varphi_{k}$ and choose an arbitrary sequence $C_{k}$ of positive reals increasing to $+\infty$, and a sequence of rationals $\delta_{k}$ decreasing to 0 . Now observe that $U_{k}:=\left\{\varphi_{k}<-\left(C_{k}+1\right) / \delta_{k}\right\}$ is an open neighbourhood of $A_{k}$ such that we have $\varphi_{k}<\left(1-\delta_{k}\right) \varphi_{k}-C_{k}-1 / 2$ on $\bar{U}_{k}$, so that

$$
\varphi \leq \varphi_{k}<\left(1-\delta_{k}\right) \varphi_{k}-C_{k}-1 / 2 \text { on the compact } \bar{U}_{k} \text {. }
$$

Thus $(a)$ and the continuity of $\psi_{j}$ show that for $j_{k}$ big enough, we have $\psi_{j_{k}}<\left(1-\delta_{k}\right) \varphi_{k}-C_{k}-1 / 2$ on $\bar{U}_{k}$. Now we select a smaller open neighbourhood $W_{k} \subset \subset U_{k}$ of $A_{k}$, and we set:

$$
\theta_{k}:= \begin{cases}\left(1-\delta_{k}\right) \varphi_{k}-C_{k} & \text { on } W_{k}, \\ \max _{\eta}\left(\left(1-\delta_{k}\right) \varphi_{k}-C_{k}, \psi_{j_{k}}\right) & \text { on } X-W_{k}\end{cases}
$$

with $\max _{\eta}(x, y)$ denoting a regularized maximum function.

If $\eta$ is chosen so small that $\max _{\eta}(x, y)=x$ when $y<x-1 / 2$ then the two parts to be glued coincide on some neighbourhood of $\partial W_{k}$, so that $\theta_{k}$ is almost plurisubharmonic with algebraic singularities on $X$.

The gluing property of plurisubharmonic functions shows that $\alpha+d d^{c} \theta_{k}$ will have a lower bound going to 0 for $k \rightarrow+\infty$ if we can show that this is the case for $\alpha+d d^{c} \psi_{j_{k}}$ on $X-W_{k}$. But we have $\nu\left(\varphi_{k}, x\right)=0$ for $x$ in this set, thus (iii) and (b) together give the result.

Now we have to see that $\left(\alpha+d d^{c} \theta_{k}\right)_{a c}(x) \rightarrow T_{a c}(x)$ almost everywhere. To do this, notice that if $\varphi(x)>-\infty$, then $x$ cannot be in $U_{k}$ for $k$ big enough, since otherwise $\varphi_{k}(x) \leq-\left(C_{k}+1\right)$ for $k$ big enough, which would yield $\varphi(x)=-\infty$ by $(i i)$. Furthermore we have

$$
\left(1-\delta_{k}\right) \varphi_{k}(x)-C_{k}<\varphi(x)-1 / 2 \leq \psi_{j}-1 / 2
$$


for $k$ big enough (since $C_{k} \rightarrow+\infty$ ) and $j$ arbitrary (by $(a)$ ), thus in particular for $j=j_{k}$. Continuity then gives $\left(1-\delta_{k}\right) \varphi_{k}-C_{k}<\psi_{j_{k}}-1 / 2$ on some neighbourhood of $x$ (depending on $k$ ) contained in $X-W_{k}$, so that $\theta_{k}=\psi_{j_{k}}$ on this neighbourhood. Thus for every $x$ outside the polar set of $\varphi$ (which has measure 0$)$ we have $\left(\alpha+d d^{c} \theta_{k}\right)(x)=S_{j_{k}}(x)$ for $k$ big enough, and the result follows by $(c)$.

To conclude this section, let us illustrate the above ideas by reproducing the following result from [DP01]:

Proposition 2.8 A compact complex manifold $X$ is in the Fujiki class $\mathcal{C}$ if, and only if, it carries a Kähler current.

Recall that $X$ is said to be in the Fujiki class $\mathcal{C}$ if it can be modified into a Kähler manifold. Since the push-forward of a Kähler form by a modification is a Kähler current, one direction is clear. Assume now that the compact complex manifold $X$ carries a Kähler current $T \geq \omega$ for some Hermitian form $\omega$. Using the above approximation result, one can assume that $T$ has analytic singularities. By corollary 2.4 , we get a finite sequence of blowingups $\mu: \widetilde{X} \rightarrow X$ such that $\mu^{\star} T$ decomposes into a sum $\alpha+D$, with $\alpha$ a smooth form and $D$ a real effective divisor. Then $\alpha \geq \mu^{\star} \omega$ is semi-positive, but not definite positive everywhere a priori. Here one uses the following

Lemma 2.9 Let $f: X \rightarrow Y$ be the blow-up of $Y$ along a submanifold $V$, let $\omega$ be a Hermitian form on $X$ and let $E$ denote the exceptional divisor of $f$. Then there exists a smooth closed form u cohomologous to $E$ such that $\mu^{\star} \omega-\varepsilon u$ is definite positive everywhere for $\varepsilon>0$ small enough.

To see this, recall that $\mathcal{O}_{E}(-E)$ is isomorphic to $\mathcal{O}_{P\left(N_{V / Y}\right)}(1)$ on $P\left(N_{V / Y}\right)=$ $E$, hence is relatively ample over $V$, so that if we choose some smooth form $\beta$ on $X$ cohomologous to $[E]$, there exists a smooth function $\varphi$ on $E$ such that $-\left(\beta_{\mid E}+d d^{c} \varphi\right)$ is definite positive along the fibers of $E \rightarrow V$. Now we extend $\varphi$ smoothly to the whole of $X$, and set $u:=\beta+d d^{c} \varphi$. If we replace $\varphi$ by $\varphi-C d(x, E)$ for $C \gg 0$, we can even arrange so that $-u$ be positive definite on $N_{E / X}$. Now we note that $\mu^{\star} \omega$ is semi-positive everywhere and strictly positive on the "horizontal" directions of $E \rightarrow V$, so that $\mu^{\star} \omega-\varepsilon u$ will be positive definite on a neighbourhood $E$ for $\varepsilon>0$ small enough. There remains to choose $\varepsilon>0$ even smaller to ensure that $\mu^{\star} \omega-\varepsilon u$ be positive outside this neighbourhood, which can be done because $\mu^{\star} \omega$ has a uniform strictly positive lower bound there.

A repeated application of this lemma will therefore yield a smooth closed form $v$ cohomologous to an effective linear combination with small coefficients of the exceptional divisors of $\mu$ such that $\alpha-v$ is definite positive, hence a Kähler form, for $\varepsilon>0$ small enough. 


\subsection{Boundedness of the mass.}

Here we are interested in the control of $\int_{X} T_{a c}^{n}$ for a closed positive $(1,1)$ current. It is by no means clear that such an integral is convergent in general, and we show that things go well in the Kähler case.

Lemma 2.10 Let $T$ be any closed positive $(1,1)$-current on $X$ compact complex. Then the Lelong numbers $\nu(T, x)$ of $T$ can be bounded by a constant depending only on the $d d^{c}$-cohomology class of $T$.

Indeed for $\omega$ a Gauduchon form on $X$ (i.e. a Hermitian form with $d d^{c}\left(\omega^{n-1}\right)=$ 0 ), one has by definition that $\nu(T, x)$ is (up to a constant depending on $\omega$ near $x$ ) the limit for $r \rightarrow 0_{+}$of

$$
\nu(T, x, r):=\frac{(n-1) !}{\left(\pi r^{2}\right)^{n-1}} \int_{B(x, r)} T \wedge \omega^{n-1},
$$

known to be an increasing function of $r$. Thus if we choose $r_{0}$ small enough to ensure that each ball $B\left(x, r_{0}\right)$ is contained in a coordinate chart, we get $\nu(T, x) \leq \nu\left(T, x, r_{0}\right) \leq C \int_{X} T \wedge \omega^{n-1}$, a quantity depending only on the $d d^{c}$ cohomology class $\{T\}$.

Lemma 2.11 (Uniform boundedness of the mass)) Let $T$ be a closed positive $(1,1)$-current on $(X, \omega)$ compact Kähler. Then the integrals $\int_{X} T_{a c}^{k} \wedge$ $\omega^{n-k}$ are finite for each $k=0, \ldots, n$ and can be bounded in terms of $\omega$ and the cohomology class of $T$ only.

Proof: By [Dem82], there exists a sequence $T_{j}$ of smooth $(1,1)$-forms in the same class as $T$ and a constant $C>0$ depending only on $(X, \omega)$ such that:

(i) $T_{j} \rightarrow T$ weakly,

(ii) $T_{j}(x) \rightarrow T_{a c}(x)$ almost everywhere, and

(iii) $T_{j} \geq-C \lambda_{j} \omega$ for some continuous functions $\lambda_{j}$ such that $\lambda_{j}(x)$ decreases to $\nu(T, x)$ when $j \rightarrow+\infty$, for every $x$.

By using the preceding lemma, one can thus find a constant also denoted by $C$ and depending on $\omega$ and the cohomology class $\{T\}$ only such that $T_{j}+C \omega \geq 0$. But now

$$
\int_{X}\left(T_{j}+C \omega\right)^{k} \wedge \omega^{n-k}=\{T+C \omega\}^{k}\{\omega\}^{n-k}
$$

does not depend on $j$, so the result follows by Fatou's lemma.

Here, even though the approximation result of Demailly does not need the Kähler assumption, the argument definitely fails in its last part without it. However it still holds true if $\operatorname{dim} X \leq 2$, as one sees in the above proof. 


\section{Volume of a line bundle}

\subsection{A Morse-type inequality}

In this part, we will prove the following:

Proposition 3.1 For L pseudoeffective on $X$ compact Kähler and $T \in c_{1}(L)$ positive one has:

$$
v(L) \geq \int_{X} T_{a c}^{n}
$$

In order to get this, we will appeal to the singular holomorphic Morse inequalities, but to state this result we first need some terminology.

(i) The Nadel multiplier ideal sheaf $\mathcal{I}(T)$ of an almost positive closed $(1,1)$ current $T$ is defined as the sheaf of germs of holomorphic functions $f$ such that $|f|^{2} e^{-2 \varphi}$ is locally integrable, for some (hence any) $\varphi \in L_{l o c}^{1}$ such $T=d d^{c} \varphi$ locally. This sheaf is coherent, as is well known (cf. e.g. [Dem96]).

(ii) The $q$-index set of an almost positive closed $(1,1)$-current $T$ is the set of $x \in X$ such that the absolutely continuous part $T_{a c}$ of $T$ has exactly $q$ negative eigenvalues at $x$. This set is denoted by $X(T, q)$, and we also write $X(T, \leq q)$ for the union of the $X(T, j)$ 's for $j=0, \ldots q$. These sets are only defined up to a null measure set, but since we shall only integrate absolutely continuous forms on them, it is not really annoying here.

Now we can state the following result, due to Bonavero [Bon93]:

Theorem 3.2 (Singular Morse inequalities) Let $L$ be any holomorphic line bundle on the compact complex $n$-dimensional manifold $X$, and $T \in$ $c_{1}(L)$ be some closed $(1,1)$-current with algebraic singularities. Then the following holds:

$$
h^{0}(X, \mathcal{O}(k L) \otimes \mathcal{I}(k T))-h^{1}(X, \mathcal{O}(k L) \otimes \mathcal{I}(k T)) \geq \frac{k^{n}}{n !} \int_{X(T, \leq 1)} T_{a c}^{n}-o\left(k^{n}\right)
$$

for $k \rightarrow+\infty$.

Since one has

$$
\begin{gathered}
h^{0}(X, \mathcal{O}(k L)) \geq h^{0}(X, \mathcal{O}(k L) \otimes \mathcal{I}(k T)) \\
\geq h^{0}(X, \mathcal{O}(k L) \otimes \mathcal{I}(k T))-h^{1}(X, \mathcal{O}(k L) \otimes \mathcal{I}(k T))
\end{gathered}
$$

Theorem 3.2 implies:

Corollary 3.3 For any line bundle $L$ on the compact complex $X$, one has

$$
v(L) \geq \int_{X(T, \leq 1)} T_{a c}^{n}
$$

for every $T \in c_{1}(L)$ with algebraic singularities. 
We can now conclude the proof of Proposition 3.1. In fact, we choose a sequence $T_{k}$ of currents with algebraic singularities as in Corollary 2.7 (here $\gamma=0)$, and we denote by $\lambda_{1} \leq \ldots \leq \lambda_{n}\left(\right.$ resp. $\left.\quad \lambda_{1}^{(k)} \leq \ldots \leq \lambda_{n}^{(k)}\right)$ the eigenvalues of $T_{a c}$ (resp. $T_{k, a c}$ ) with respect to $\omega$. We have by assumption that $\lambda_{1} \geq 0, \lambda_{1}^{(k)} \geq-\varepsilon_{k}$ and $\lambda_{j}^{(k)}(x) \rightarrow \lambda_{j}(x)$ almost everywhere. We can certainly assume that $\int_{X} T_{a c}^{n}>0$, which means that the set $A:=\left\{\lambda_{1}>0\right\}$ has positive measure. For each small $\delta>0$, Egoroff's lemma gives us some $B_{\delta} \subset A$ such that $\lambda_{1}^{(k)} \rightarrow \lambda_{1}$ uniformly on $B_{\delta}$ and also $A-B_{\delta}$ has measure less than $\delta$. Thus we see that $B_{\delta} \subset X\left(T_{k}, 0\right)$ for $k$ big enough, and consequently $\limsup \int_{X\left(T_{k}, 0\right)} T_{k, a c}^{n} \geq \int_{B_{\delta}} \liminf T_{k, a c}^{n}=\int_{B_{\delta}} T_{a c}^{n}$, using Fatou's lemma. Letting now $\delta$ tend to 0 , we get

$$
\limsup \int_{X\left(T_{k}, 0\right)} T_{k, a c}^{n} \geq \int_{A} T_{a c}^{n}=\int_{X} T_{a c}^{n}
$$

Since by Corollary 3.3 above we have $\int_{X\left(T_{k}, 0\right)} T_{k, a c}^{n} \leq v(L)-\int_{X\left(T_{k}, 1\right)} T_{k, a c}^{n}$ for every $k$, the proof of the inequality will be over if we can show that

$-\int_{X\left(T_{k}, 1\right)} T_{k, a c}^{n} \rightarrow 0$. But we observe the following inequalities on $X\left(T_{k}, 1\right)$ :

$$
0 \leq-T_{k, a c} \leq n \varepsilon_{k} \omega \wedge\left(T_{k, a c}+\varepsilon_{k} \omega\right)^{n-1},
$$

from which we get

$$
0 \leq-\int_{X\left(T_{k}, 1\right)} T_{k, a c}^{n} \leq n \varepsilon_{k} \int_{X} \omega \wedge\left(T_{k, a c}+\varepsilon_{k} \omega\right)^{n-1} .
$$

Now the last integral is bounded uniformly in terms of $\{T\}$ and $\omega$ only by the "boundedness of the mass" (Lemma 2.11), which ends the proof.

It is worth noting that the Kähler assumption is needed precisely for this last lemma. Consequently, Proposition 3.1 is also true on any surface.

\subsection{The theorem of Calabi-Yau.}

We fix a Kähler form $\omega$ with $\int_{X} \omega^{n}=1$. We seek a positive $T$ in $c_{1}(L)$ such that $T_{a c}^{n} \geq v(L) \omega^{n}(\star)$. If this is done, the proof of Theorem 1.2 is over, since the fact that $\int_{X} T_{a c}^{n} \leq \int_{X} v(L) \omega^{n}$ (by Proposition 3.1) implies that ( $\star$ ) is an equality a.e. First let us recall the fundamental result proved in [Yau78]:

Theorem 3.4 (Aubin-Calabi-Yau) Let $(X, \omega)$ be a compact Kähler manifold, and assume that $\int_{X} \omega^{n}=1$. Then given a Kähler cohomology class a, there exists a Kähler form $\alpha \in$ a such that

$$
\alpha^{n}=\left(\int_{X} a^{n}\right) \omega^{n}
$$

pointwise. 
We now appeal to Fujita's Theorem 1.1, and use the notations in it. Notice first that if our $L$ is not big, we will have $0=v(L) \geq \int_{X} T_{a c}^{n} \geq 0$ for every positive $T \in c_{1}(L)$ because of what we have already seen, so that any such $T$ would do. We thus assume that $L$ is big, which implies automatically the projectivity of $X$ since the latter will be Kähler and Moishezon at the same time.

Now we choose any Kähler form $\omega_{\varepsilon}$ on $X_{\varepsilon}$, and apply the Calabi-Yau theorem to $A_{\varepsilon}$ with respect to the Kähler form $\mu_{\varepsilon}^{\star} \omega+\delta \omega_{\varepsilon}$, normalized adequately, and we get a Kähler form $\alpha_{\varepsilon, \delta} \in c_{1}\left(A_{\varepsilon}\right)$ such that

$$
\alpha_{\varepsilon, \delta}^{n}=\frac{v\left(A_{\varepsilon}\right)}{\int_{X_{\varepsilon}}\left(\mu_{\varepsilon}^{\star} \omega+\delta \omega_{\varepsilon}\right)^{n}}\left(\mu_{\varepsilon}^{\star} \omega+\delta \omega_{\varepsilon}\right)^{n} .
$$

Now consider

$$
T_{\varepsilon, \delta}:=\left(\mu_{\varepsilon}\right)_{\star}\left(\alpha_{\varepsilon, \delta}+\left[E_{\varepsilon}\right]\right) .
$$

This is a positive current lying in $c_{1}(L)$, and so standard compactness properties of currents show that for each $\varepsilon$ there exists some sequence $\delta_{k}(\varepsilon)$ decreasing to 0 such that $T_{\varepsilon, \delta_{k}}$ converges to some positive $T_{\varepsilon} \in c_{1}(L)$. For the same reason there exists a sequence $\varepsilon_{k}$ decreasing to 0 such that $T_{\varepsilon_{k}}$ converges to some positive $T \in c_{1}(L)$. We will show that this $T$ satisfies $T_{a c}^{n} \geq v(L) \omega^{n}$ almost everywhere, which will conclude the proof. This will be handled by the following semicontinuity property:

Lemma 3.5 Let $T_{k}$ be a sequence of positive $(1,1)$-currents converging weakly to $T$. Then one has $T_{a c}^{n} \geq \lim \sup T_{k, a c}^{n}$ a.e.

Proof: We fix a $\omega$ a Hermitian form, and for $\alpha$ a positive (1,1)-form we denote by $\operatorname{det}(\alpha)$ the determinant of $\alpha$ with respect to $\omega$, that is $\operatorname{det}(\alpha)=$ $\alpha^{n} / \omega^{n}$. Since the result is local, we may consider a regularizing sequence $\left(\rho_{j}\right)$. The concavity of the function $A \rightarrow \operatorname{det}(A)^{1 / n}$ on the convex cone of Hermitian semi-positive matrices of size $n$ then yields the second of the following inequalities

$$
\operatorname{det}\left(T_{k} \star \rho_{j}\right)^{1 / n} \geq \operatorname{det}\left(T_{k, a c} \star \rho_{j}\right)^{1 / n} \geq \operatorname{det}\left(T_{k, a c}\right)^{1 / n} \star \rho_{j} .
$$

Since convolution transforms a weak convergence into a $C^{\infty}$ one, Fatou's lemma therefore implies:

$$
\operatorname{det}\left(T \star \rho_{j}\right)^{1 / n} \geq\left(\liminf \operatorname{det}\left(T_{k, a c}\right)^{1 / n}\right) \star \rho_{j} .
$$

Now Lebesgue's theorem implies that $T \star \rho_{j} \rightarrow T_{a c}$ a.e., thus we get

$$
\operatorname{det}\left(T_{a c}\right)^{1 / n} \geq \liminf \operatorname{det}\left(T_{k, a c}\right)^{1 / n},
$$


. We can eventually turn the lim inf into a lim sup by choosing subsequences pointwise.

Remark: this type of arguments (Calabi-Yau+convolution and concavity) can already be found in [Dem93].

\section{Volume of a pseudoeffective class.}

\subsection{General properties.}

In this section, $X$ is a compact Kähler manifold unless otherwise specified.We propose to extend some results related to the volume of a line bundle, that is to say of an entire (or even rational) pseudoeffective class, to the more general case of an arbitrary pseudoeffective $(1,1)$-class $a$. Note that the volume $v(a)$ of $a$, as defined in the introduction, is always finite by Lemma 2.11 (boundedness of the mass).

First of all, let us state the following

Proposition 4.1 If $f: X \rightarrow Y$ is a generically finite holomorphic map between compact Kähler manifolds and $a$ (resp. $b$ ) is a pseudoeffective $(1,1)$ class on $X($ resp. $Y)$, then one has $v\left(f_{\star} a\right) \geq v(a)\left(\right.$ resp. $\left.v\left(f^{\star} b\right)=(\operatorname{deg} f) v(b)\right)$.

This is consequence of the following easy facts:

Lemma 4.2 Let $f$ be as above. Then the following properties hold:

(i) If $\alpha$ is a form with $L_{l o c}^{1}$ coefficients on $X$, then $f_{\star} \alpha$ (taken pointwise) is $L_{l o c}^{1}$ on $Y$ (this only requires $f$ to be proper and surjective), and $f_{\star}\left(\alpha^{k}\right)=\left(f_{\star} \alpha\right)^{k}$ (taken pointwise) whenever $\alpha^{k}$ is also $L_{l o c}^{1}$,

(ii) If $\omega$ is an integrable top degree form on $X$ (resp. on $Y$ ), then $\int_{Y} f_{\star} \omega=$ $\int_{X} \omega\left(\right.$ resp. $\left.\int_{X} f^{\star} \omega=(\operatorname{deg} f) \int_{Y} \omega\right)$,

(iii) If $T$ is a positive $(1,1)$-current on $X$ (resp. on $Y)$, then $\left(f_{\star} T\right)_{a c}=f_{\star}\left(T_{a c}\right)$ a.e. (resp. $\left(f^{\star} T\right)_{a c}=f^{\star}\left(T_{a c}\right)$ a.e. $)$ where $f_{\star}\left(T_{a c}\right)\left(\right.$ resp. $\left.f^{\star}\left(T_{a c}\right)\right)$ are meant pointwise.

We have already seen that for a nef line bundle $L$ one has $v(L)=L^{n}$. This remains true for an arbitrary nef class:

Proposition 4.3 Let a be a nef class on $X$. Then one has

$$
v(a)=\int_{X} a^{n}
$$

The proof is in two steps. First we give the following more precise nef version of the boundedness-of-the-mass lemma, due to Mourougane [Mou98]: 
Lemma 4.4 Given a nef class $a$ on the Kähler manifold $(X, \omega)$ (or any compact complex surface), one has for every positive $T \in$ a the following inequalities:

$$
\int_{X} T_{a c}^{k} \wedge \omega^{n-k} \leq \int_{X} a^{k} \wedge \omega^{n-k}
$$

for $k=0, \ldots, n$.

For the proof, we write as before $T=\alpha+d d^{c} \varphi$ with $\alpha$ smooth in $a$, and consider a smooth sequence $T_{j}=\alpha+d d^{c} \psi_{j}$ in $a$ such that

(i) $\psi_{j}$ decreases to $\varphi$,

(ii) $T_{j} \geq-C \lambda_{j} \omega$ for $C>0$ and $\lambda_{j}$ continuous decreasing to the Lelong number,

(iii) $\left(T_{j}\right)(x) \rightarrow T_{a c}(x)$ almost everywhere.

Since $a$ is nef, we also have by definition a sequence of smooth forms $\alpha_{k}=$ $\alpha+d d^{c} \varphi_{k}$ such that $\alpha_{k} \geq-\varepsilon_{k} \omega$ for a sequence $\varepsilon_{k}$ decreasing to 0 . We will again glue these two sequences together to get a smooth sequence in $a$ with small negative part and a good behaviour with respect to the absolutely continuous part, and then the same argument as for the boundedness of the mass will allow us to conclude.

In fact, we take a sequence of positive reals $C_{k}$ converging to $+\infty$, and we set $\theta_{k, j}:=\max _{\eta}\left(\varphi_{k}-C_{k}, \psi_{j}\right)$. We will show that for each $k$ we can choose $j_{k}$ big enough so that $\theta_{k}:=\theta_{k, j_{k}}$ has a Hessian admitting a lower bound going to 0 . By (ii), it is enough to show that given $\delta>0$ and $k$, we have $\psi_{j}<\varphi_{k}-\left(C_{k}+1\right)$ near $E_{\delta}(T)$ for $j$ big enough. But since $\varphi$ has poles on the compact set $E_{\delta}(T)$, it is just a consequence of $(i)$ and the continuity of $\psi_{j}$.

As a consequence of this lemma, we get of course that $v(a) \leq \int_{X} a^{n}$ for $a$ nef. To get the converse inequality, we will prove the following result analogous to Theorem 1.2:

Proposition 4.5 (Calabi-Yau, nef case) Given a Kähler form $\omega$ with $\int_{X} \omega^{n}=1$, there exists a positive $T \in$ a such that

$$
T_{a c}^{n}=\left(\int_{X} a^{n}\right) \omega^{n}
$$

almost everywhere.

This is of course a consequence of the Calabi-Yau theorem as follows: for every $\varepsilon>0$ there exists a Kähler form $\alpha_{\varepsilon} \in a+\varepsilon \omega$ such that

$$
\alpha_{\varepsilon}=\left(\int_{X}(a+\varepsilon \omega)^{n}\right) \omega^{n}
$$


pointwise. Since the mass $\int_{X} \alpha_{\varepsilon} \wedge \omega^{n-1}=\{a+\varepsilon \omega\}\{\omega\}^{n-1}$ is bounded, there exists some weak limit $T \in a$ of $\alpha_{\varepsilon}$. Then Lemma 3.5 yields $T_{a c}^{n} \geq\left(\int_{X} a^{n}\right) \omega^{n}$. By the reverse inequality proven above, this is enough to conclude.

\subsection{A degenerate Calabi-Yau theorem.}

In this section, we prove the following more general version of Theorem 1.2:

Theorem 4.6 (Calabi-Yau, pseudoeffective case) If a is a pseudoeffective class and $\omega$ a Kähler form with $\int_{X} \omega^{n}=1$, then there is a positive $T \in a$ such that

$$
T_{a c}^{n}=v(a) \omega^{n}
$$

almost everywhere.

Proof: choose a positive $T \in a$ with $\int_{X} T_{a c}^{n}$ close to $v(a)$, and then a sequence $T_{\varepsilon} \in a$ with analytic singularities such that $T_{\varepsilon} \geq-\varepsilon \omega$ and $T_{\varepsilon, a c} \rightarrow T_{a c}$ almost everywhere. Let then $\mu_{\varepsilon}: X_{\varepsilon} \rightarrow X$ be a modification such that $\mu_{\varepsilon}^{\star} T_{\varepsilon}$ decomposes as a sum $\alpha_{\varepsilon}+D_{\varepsilon}$ with $\alpha_{\varepsilon}$ smooth and $D_{\varepsilon}$ a real effective divisor. Then $\alpha_{\varepsilon}+\varepsilon \mu_{\varepsilon}^{\star} \omega$ defines a nef class on $X_{\varepsilon}$, so by the nef case of Calabi-Yau, if we select an arbitrary Kähler form $\omega_{\varepsilon}$ on $X_{\varepsilon}$ and $\delta>0$, there is a positive $T_{\varepsilon, \delta}$ cohomologuous to $\alpha_{\varepsilon}+\varepsilon \mu_{\varepsilon}^{\star} \omega$ such that

$$
T_{\varepsilon, \delta, a c}^{n}=\frac{v\left(\alpha_{\varepsilon}+\varepsilon \mu_{\varepsilon}^{\star} \omega\right)}{v\left(\mu_{\varepsilon}^{\star} \omega+\delta \omega_{\varepsilon}\right)}\left(\mu_{\varepsilon}^{\star} \omega+\delta \omega_{\varepsilon}\right)^{n}
$$

almost everywhere. Now we look at the current

$$
S_{\varepsilon, \delta}:=\mu_{\varepsilon, \star}\left(T_{\varepsilon, \delta}+D_{\varepsilon}\right)
$$

on $X$. Its cohomology class is $a+\varepsilon \omega$, so after extracting sequences decreasing to 0 for $\delta, \varepsilon$ in that order we get some current

$$
S=\lim _{\varepsilon \rightarrow 0} \lim _{\delta \rightarrow 0} S_{\varepsilon, \delta}
$$

in the class $a$. Since $\mu_{\varepsilon}$ is a local isomorphism almost everywhere, we have

$$
S_{\varepsilon, \delta, a c}^{n}=\frac{v\left(\alpha_{\varepsilon}+\varepsilon \mu_{\varepsilon}^{\star} \omega\right)}{v\left(\mu_{\varepsilon}^{\star} \omega+\delta \omega_{\varepsilon}\right)}\left(\omega+\delta \mu_{\varepsilon, \star} \omega_{\varepsilon}\right)^{n}
$$

almost everywhere, and Lemma 3.5 therefore implies:

$$
S_{a c}^{n} \geq\left(\lim _{\varepsilon \rightarrow 0} \int_{X}\left(T_{\varepsilon, a c}+\varepsilon \omega\right)^{n}\right) \omega^{n}
$$


The inner limit is greater than $\int_{X} T_{a c}^{n}$ by Fatou's lemma. Now we let $\int_{X} T_{a c}^{n}$ tend to $v(a)$, and choose $S_{\infty}$ some accumulation point of the currents $S$ obtained above, so that eventually we obtain $S_{\infty, a c}^{n} \geq v(a) \omega^{n}$. By definition of $v(a)$, this inequality is in fact an equality (almost everywhere), which concludes the proof.

Let us summarize now some properties of the volume:

Proposition 4.7 The function $a \rightarrow v(a)$ defined on the pseudoeffective cone is homogeneous of degree $n$, it satisfies $v(a+b) \geq v(a)+v(b)$ and it is continuous. Furthermore its restriction to the nef cone satisfies $v(a)=a^{n}$.

Homogeneity and superadditivity being trivial, only the continuity needs a proof. But a consequence of the previous proof is the second of the inequalities $v(a) \leq \lim _{\varepsilon \rightarrow 0} v(a+\varepsilon \omega) \leq v(a)$, which easily implies the result.

\subsection{The Grauert-Riemenschneider criterion.}

We have seen in the first section that for a pseudoeffective line bundle $L$ on a compact Kähler manifold, the existence of a positive curvature current $T$ in $c_{1}(L)$ with $\int_{X} T_{a c}^{n}>0$ implies that $L$ is big. This is a GrauertRiemenschneider-type criterion for bigness, which we would like to extend to any (i.e. not necessarily rational) pseudoeffective class. As a matter of fact, let us say that a $\left(d d^{c}\right)$-cohomology $(1,1)$-class is big if it contains a Kähler current. Then we prove the

Theorem 4.8 (Grauert-Riemenschneider criterion, Kähler case) If a is a pseudoeffective $(1,1)$-class on the compact Kähler manifold $X$, then a is big if, and only if, $v(a)>0$.

This has been proven for a nef class by Demailly and Paun [DP01] as an essential step for their Nakai-Moishezon criterion for Kähler classes. Namely, they proved the

Lemma 4.9 If $a$ is a nef class $X$ with $\int_{X} a^{n}>0$, then for every irreducible analytic subset $Y$ of $X$ of codimension $p$, there is some $\delta>0$ such that $a^{p} \geq \delta[Y]$, with $\geq$ meaning "more pseudoeffective than".

Once this is proven, the following quite tricky argument also due to [DP01] enables us to conclude.

Denote by $p_{1}, p_{2}$ the two projections $\widetilde{X}:=X \times X \rightarrow X$, and by $\Delta$ the diagonal. If $a$ is nef on $X$, then $\widetilde{a}=p_{1}^{\star} a+p_{2}^{\star} a$ is nef on $\widetilde{X}$, and it is easy to 
see that $\int_{\widetilde{X}} \widetilde{a}^{2 n}=\left(\begin{array}{c}2 n \\ n\end{array}\right)\left(\int_{X} a^{n}\right)^{2}>0$, so that by the above proposition one has for some $\delta>0$ : $\widetilde{a}^{n} \geq \delta[\Delta]$.

But then for $\omega$ a Kähler form on $X$ we get: $p_{1, \star}\left(\widetilde{a}^{n} \wedge p_{2}^{\star} \omega\right) \geq \delta p_{1, \star}\left([\Delta] \wedge p_{2}^{\star} \omega\right)$. The first term in the inequality is easily seen to be $n\left(\int_{X} a^{n-1} \wedge \omega\right) a$, whereas the second is $\delta \omega$, whence we eventually get $a \geq\left(\delta /\left(n \int_{X} a^{n-1} \wedge \omega\right)\right) \omega$, which is the expected conclusion.

What we plan to do is to apply the above strategy to the "nef parts" of "approximate Zariski decompositions" of $a$. More concretely, reasoning as above, we can choose a sequence $T_{k} \in a$ of currents with analytic singularities such that $T_{k} \geq-\varepsilon_{k} \omega$ and also that $v\left(T_{k, a c}+\varepsilon_{k} \omega\right)$ is bounded away from zero. Then we consider finite sequences of blowing-ups $\mu_{k}: X_{k} \rightarrow X$ such that $\mu_{k}^{\star} T_{k}=\alpha_{k}+D_{k}$ with $\alpha_{k}$ smooth and $D_{k}$ a real effective divisor. Then the class $b_{k}:=\left\{\alpha_{k}+\varepsilon_{k} \mu_{k}^{\star} \omega\right\}$ is nef and has volume bounded away from zero. Denote by $\widetilde{X}_{k}$ the product $X_{k} \times X_{k}$, by $p_{1}$ and $p_{2}$ the two projections, and $\Delta_{k}$ the diagonal. Consider moreover the class $\widetilde{b}_{k}:=p_{1}^{\star} b_{k}+p_{2}^{\star} b_{k}$. Then $\widetilde{b}_{k}$ is nef, and has volume $v\left(\widetilde{b}_{k}\right)=\left(\begin{array}{c}2 n \\ n\end{array}\right) v\left(b_{k}\right)^{2}$ uniformly bounded away from zero. We claim the following

Lemma 4.10 There exists $\delta>0$ such that $\widetilde{b}_{k}^{n} \geq \delta\left[\Delta_{k}\right]$ for every $k$.

Assuming for the moment that the lemma holds true, choose by means of Lemma 2.9 a sequence of smooth forms $u_{k}$ cohomologous to an effective linear combination $E_{k}$ of exceptional divisors of $\mu_{k}$ uch that $\omega_{k}:=\mu_{k}^{\star} \omega-\eta_{k} u_{k}$ is a Kähler form for every $k$. Then Lemma 4.10 implies that $p_{1, \star}\left(\widetilde{b}_{k}^{n} \wedge p_{2}^{\star} \omega_{k}\right) \geq$ $p_{1, \star}\left(\delta\left[\Delta_{k}\right] \wedge p_{2}^{\star} \omega_{k}\right)$ on $X_{k}$. But the left hand side is $n\left(\int_{X_{k}} b_{k}^{n-1} \wedge \omega_{k}\right) b_{k} \leq$ $n\left(\int_{X_{k}} b_{k}^{n-1} \wedge \omega_{k}\right) \mu_{k}^{\star}\left(a+\varepsilon_{k} \omega\right)$ and the right hand side is $\delta \omega_{k}$, thus pushing this forward by $\mu_{k}$, we eventually get

$$
n\left(\int_{X_{k}} b_{k}^{n-1} \wedge \omega_{k}\right)\left(a+\varepsilon_{k} \omega\right) \geq \delta \omega
$$

on $X$. It remains to notice that

$$
\begin{gathered}
\int_{X_{k}} b_{k}^{n-1} \wedge \omega_{k}=\left\{b_{k}\right\}^{n-1}\left\{\mu_{k}^{\star} \omega-\left[E_{k}\right]\right\} \\
\leq \int_{X} b_{k}^{n-1} \wedge \mu_{k}^{\star} \omega=\int_{X}\left(\mu_{k, \star} \alpha_{k}+\varepsilon_{k} \omega\right)^{n-1} \wedge \omega
\end{gathered}
$$

(the inequality holds for $b_{k}$ is nef); now the class $\left\{\mu_{k, \star} \alpha_{k}+\varepsilon_{k} \omega\right\}$ is bounded, thus we have a uniform bound for the integrals $\int_{X_{k}} b_{k}^{n-1} \wedge \omega_{k}$, and this is enough to conclude.

It remains to prove the above lemma. To do this we use the following results from [DP01]: 
Lemma 4.11 (Concentration of the mass) Given a Kähler form $\omega$ on $X$ and $Y$ an analytic subset, there exists an almost plurisubharmonic function $\varphi$ on $X$ and a sequence $\varphi_{\varepsilon}$ of smooth functions decreasing pointwise to $\varphi$ such that if we set $\omega_{\varepsilon}:=\omega+d d^{c} \varphi_{\varepsilon}$ then:

(i) $\varphi$ has analytic singularities along $Y$,

(ii) $\omega_{\varepsilon} \geq \frac{1}{2} \omega$

(iii) For any smooth point $x \in Y$ at which $Y$ is p-codimensional and every neighbourhood $U$ of $x$, there exists $\delta_{U}>0$ such that $\int_{U \cap V_{\varepsilon}} \omega_{\varepsilon}^{p} \wedge \omega^{n-p} \geq \delta_{U}$ for every $\varepsilon$, if $V_{\varepsilon}=\{\varphi<\log \varepsilon\}$.

Lemma 4.12 Let $(X, \omega)$ be compact Kähler. Given an analytic subset $Y$, a function $\varphi$ and a family of closed smooth forms $\omega_{\varepsilon}$ cohomologous to $\omega$ such that (i), (ii) and (iii) in the above lemma hold, then for each nef class a on $X$ such that $v(a)>0$, there exists $T \in a^{p}$ a closed positive current with

$$
\int_{U \cap Y} T \wedge \omega^{n-p} \geq \eta
$$

where $\eta=C v(a) \delta^{2} / M v(\omega)>0$ with $C$ a universal constant, $M=\int_{X} a^{n-p} \wedge$ $\omega^{p}$ and $\delta:=\delta_{U}$.

The proof of 4.12 goes as follows: for each $\varepsilon>0$, there exists by the CalabiYau theorem a smooth Kähler form $\alpha_{\varepsilon}$ in the class $a+\varepsilon \omega$ such that

$$
\alpha_{\varepsilon}^{n}=\frac{v(a+\varepsilon \omega)}{v(\omega)} \omega_{\varepsilon}^{n} .
$$

Denote by $\lambda_{1}^{\varepsilon} \leq \ldots \leq \lambda_{n}^{\varepsilon}$ the eigenvalues of $\alpha_{\varepsilon}$ with respect to $\omega_{\varepsilon}$. Then we find:

(a) $\lambda_{1}^{\varepsilon} \ldots \lambda_{n}^{\varepsilon}=v(a+\varepsilon \omega) / v(\omega)$.

(b) $\alpha_{\varepsilon}^{p} \geq \lambda_{1}^{\varepsilon} \ldots \lambda_{p}^{\varepsilon} \omega_{\varepsilon}^{p}$.

(c) $\alpha_{\varepsilon}^{n-p} \wedge \omega_{\varepsilon}^{p} \geq \frac{p !(n-p) !}{n !} \lambda_{p+1}^{\varepsilon} \ldots \lambda_{n}^{\varepsilon} \omega_{\varepsilon}^{n}$.

By $(c)$, we get

$$
\int_{X} \lambda_{p+1}^{\varepsilon} \ldots \lambda_{n}^{\varepsilon} \omega_{\varepsilon}^{n} \leq\left(\begin{array}{c}
n \\
p
\end{array}\right)\{a+\varepsilon \omega\}^{n-p}\{\omega\}^{p}=: M_{\varepsilon}
$$

thus in particular the set $E_{\eta}:=\left\{\lambda_{p+1}^{\varepsilon} \ldots \lambda_{n}^{\varepsilon} \geq M_{\varepsilon} / \eta\right\}$ has $\int_{E_{\eta}} \omega_{\varepsilon}^{n} \leq \eta$ for $\eta>0$ small enough. Now $(a)$ and $(b)$ yield

$$
\int_{U \cap V_{\varepsilon}} \alpha_{\varepsilon}^{p} \wedge \omega^{n-p} \geq \frac{v(a+\varepsilon \omega)}{v(\omega)} \int_{U \cap V_{\varepsilon}} \frac{1}{\lambda_{p+1}^{\varepsilon} \ldots \lambda_{n}^{\varepsilon}} \omega_{\varepsilon}^{p} \wedge \omega^{n-p}
$$




$$
\geq \frac{v(a+\varepsilon \omega)}{v(\omega)} \int_{U \cap V_{\varepsilon}-E_{\eta}} \frac{\eta}{M_{\varepsilon}} \omega_{\varepsilon}^{p} \wedge \omega^{n-p} .
$$

Observe that

$$
\int_{U \cap V_{\varepsilon}-E_{\eta}} \omega_{\varepsilon}^{p} \wedge \omega^{n-p} \geq \int_{U \cap V_{\varepsilon}} \omega_{\varepsilon}^{p} \wedge \omega^{n-p}-\int_{E_{\eta}} \omega_{\varepsilon}^{p} \wedge \omega^{n-p}
$$

The first integral is greater than $\delta$ by assumption (iii), and since $\omega_{\varepsilon}^{p} \wedge \omega^{n-p} \leq 2^{n-p} \omega_{\varepsilon}^{n}$ by $(i i)$, the second integral will be less than $2^{n-p} \eta$. Combining all this yields in the end

$$
\int_{U \cap V_{\varepsilon}} \alpha_{\varepsilon}^{p} \wedge \omega^{n-p} \geq \frac{v(a+\varepsilon \omega)}{v(\omega)} \frac{\eta}{M_{\varepsilon}}\left(\delta-2^{n-p} \eta\right) .
$$

Now we take $\eta:=\delta / 2^{n-p+1}$ and we choose $T$ some accumulation point of $\alpha_{\varepsilon}^{p}$. Then $T$ is a closed positive current in the cohomology class $a^{p}$ such that

$$
\int_{U \cap Y} T \wedge \omega^{n-p} \geq C \frac{v(a)}{v(\omega)} \frac{\delta^{2}}{M}
$$

which concludes the proof.

We now denote by $\widetilde{\mu}_{k}: \widetilde{X}_{k} \rightarrow \widetilde{X}$ the product map $\mu_{k} \times \mu_{k}$, and we select on each $\widetilde{X}_{k}$ some Kähler form $\widetilde{\omega}_{k}$. First we apply Lemma 4.11 to $Y=\Delta$ in $\widetilde{X}$ and some neighbourhood $U$ of some point $x \in \Delta$ chosen to be a regular value of $\widetilde{\mu}_{k}$ for each $k$, so that we get $\varphi, \omega_{\varepsilon}$ and $\delta=\delta_{U}$ as in the lemma. Then we consider for every $\rho>0$ the Kähler forms $\omega_{k, \varepsilon}:=\widetilde{\mu}_{k}^{\star} \omega_{\varepsilon}+\rho \widetilde{\omega}_{k}$ and $\omega_{k}:=\widetilde{\mu}_{k}^{\star} \omega+\rho \widetilde{\omega}_{k}$ and the function $\varphi_{k}:=\widetilde{\mu}_{k}^{\star} \varphi$ on $\widetilde{X}_{k}$. Then $(i),(i i)$ and $(i i i)$ still hold true, so that by Lemma 4.12 we get a closed positive $T_{k} \in \widetilde{b}_{k}^{n}$ such that

$$
\int_{U_{k} \cap Y_{k}} T_{k} \wedge \omega_{k}^{n} \geq \eta_{k}
$$

with $U_{k}:=\widetilde{\mu}_{k}^{-1} U, Y_{k}:=\widetilde{\mu}_{k}^{-1} Y$ and $\eta_{k}=C v\left(b_{k}\right) \delta^{2} / M_{k} v\left(\omega_{k}\right)$ for $M_{k}=\int_{\widetilde{X}_{k}} \widetilde{b}_{k}^{n} \wedge \omega_{k}^{p}$. All this was depending on $\rho$, so we can now let $\rho$ tend to zero $\left(k\right.$ is fixed) so as to get $\Theta_{k}$ some accumulation point of the $\left(T_{k}(\rho)\right)_{\rho}$ with

$$
\int_{U_{k} \cap Y_{k}} \Theta_{k} \wedge \widetilde{\mu}_{k}^{\star} \omega^{n} \geq \eta_{k}
$$

for $\eta_{k}=C v\left(b_{k}\right) \delta^{2} / M_{k} v\left(\widetilde{\mu}_{k}^{\star} \omega\right)$ and $M_{k}=\int_{\widetilde{X}_{k}} \widetilde{b}_{k}^{n} \wedge \widetilde{\mu}_{k}^{\star} \omega^{n}$. Now $M_{k}$ and $v\left(\widetilde{\mu}_{k}^{\star} \omega\right)=$ $v(\omega)$ are certainly under uniform control, thus so is $\eta_{k}$, and the above equality compels $\Theta_{k}$ to have positive mass bounded away from zero on $\Delta_{k}$, since any other irreducible component of $Y_{k}$ is exceptional with respect to $\widetilde{\mu}_{k}$. Since $\Theta_{k}$ 
has bidimension $(n, n)$ and $\Delta_{k}$ is $n$-dimensional, Siu's decomposition formula then shows that $\Theta_{k} \geq \eta\left[\Delta_{k}\right]$ for some uniform $\eta$, which at last concludes the proof.

Let us now state some immediate corollaries of this bigness criterion:

Proposition 4.13 Let $f: X \rightarrow Y$ be a generically finite holomorphic map between compact Kähler manifolds, and $a, b$ be $(1,1)$-cohomology classes on $X$ and $Y$ respectively. Then one has:

(i) a big implies $f_{\star} a$ big,

(ii) b big implies $f^{\star} b$ big.

The first point stems from the fact that pushing forward a Kähler current yields a Kähler current; as to the second, it is a consequence of Proposition 4.1 and the Grauert-Riemenschneider criterion.

Proposition 4.14 If a is a pseudoeffective class with $v(a)>0$, there exists a sequence $T_{k} \in$ a of Kähler currents with analytic singularities such that $\int_{X} T_{k, a c}^{n} \rightarrow v(a)$ as $k \rightarrow+\infty$.

Indeed, choose a positive $T \in a$ with $\int_{X} T_{a c}^{n}$ close to $v(a)$, and $T_{1} \in a$ a Kähler current. Then for each $\varepsilon>0$ small $T_{\varepsilon}:=\varepsilon T_{1}+(1-\varepsilon) T$ is a Kähler current in $a$ with $T_{\varepsilon, a c}=\varepsilon T_{1, a c}+(1-\varepsilon) T_{a c}$, thus $\int_{X} T_{\varepsilon, a c}^{n}$ can be made as close to $v(a)$ as desired. Now we can approach each $T_{\varepsilon}$ by Kähler currents with analytic singularities such that the absolutely continuous parts converge a.e., and we conclude by Fatou's lemma.

From the latter proposition, we deduce a general form of Fujita's theorem:

Theorem 4.15 (Approximation of the volume) Let a be a pseudoeffective $(1,1)$-class on $X$ compact Kähler. Assume that the volume $v(a)$ is positive. Then for each $\varepsilon>0$ small there exists a finite sequence of blowing-ups $\mu: \widetilde{X} \rightarrow X$, a Kähler class $\omega$ and a real effective divisor $D$ on $\widetilde{X}$ such that: (i) $\mu^{\star} a=\omega+D$

(ii) $v(\omega) \leq v(a) \leq v(\omega)+\varepsilon$

If a is rational, $\omega$ and $D$ can be taken to be rational.

Remark: of course the rational case is the original result of Fujita.

Proof of the theorem: select first $T \in a$ a Kähler current with analytic singularities such that $\int_{X} T_{a c}^{n}>v(a)-\varepsilon$, as the proposition above allows; then take a resolution of singularities $\mu: \widetilde{X} \rightarrow X$ of $T$, so that $\mu^{\star} T=\alpha+D$ with $\alpha$ smooth and $D$ an effective divisor. If we denote by $E$ the exceptional divisor of $\mu$, Lemma 2.9 implies that the class of $\alpha-\delta E$ is Kähler for each $\delta>0$ small enough, thus the decomposition 


$$
\mu^{\star} a=(\alpha-\delta E)+(D+\delta E)
$$

is the one sought after for $\delta>0$ small enough, since $v(\alpha-\delta E) \geq \int_{\widetilde{X}} \alpha^{n}=$ $\int_{X} T_{a c}^{n}$. When $a$ is rational, on can arrange for $D$ to be rational by a slight perturbation which can be absorbed by $\alpha$, and $\delta$ can be taken rational as well, q.e.d.

\subsection{Miscellaneous}

\subsubsection{The non-Kähler case}

Thanks to Lemma 4.2, the property of uniform boundedness of the mass is invariant under modifications, thus still holds true on any compact complex manifold in the Fujiki class $\mathcal{C}$ (a Fujiki manifold for short). As a consequence, all the results which are not an equality of Calabi-Yau type remain true when the Kähler assumption on $X$ is replaced by: $X$ is Fujiki. Since the presence of a big class on $X$ forces it to be Fujiki by Proposition 2.8, we see for instance that the general form of Fujita's theorem (Theorem 4.15) is true on an arbitrary compact complex manifold if we assume $a$ to be big instead of having positive volume. Nevertheless, it is tempting to think that the Grauert-Riemenschneider criterion holds true in general, which comes down to the following conjecture (cf. also [DP01]):

Conjecture: If a compact complex manifold $X$ carries a closed positive $(1,1)$-current $T$ with $\int_{X} T_{a c}^{n}>0$, then $X$ is Fujiki.

This conjecture is true for $\operatorname{dim} X=2$, as we will see.

\subsubsection{The case of a surface}

In this section, we assume that $X$ is a compact complex surface. Consider $T$ a closed positive $(1,1)$-current on $X$, and let $T=D+R$ stand for its Siu decomposition. Then we claim that the $d d^{c}$-class $\{R\}$ is nef, and is even Kähler when $T$ is a Kähler current. This is in fact a straightforward consequence of a result of Paun given in [DP01], which says that a pseudoeffective class (resp. a big class) $\{T\}$ is nef (resp. Kähler) if its restriction $\{T\}_{\mid Y}$ is nef (resp. Kähler) for every irreducible analytic subset contained in some Lelong sublevel set $E_{c}(T)$. We will however briefly recall the argument: let $R_{k}$ be an approximation of $R$ with analytic singularities as in Theorem 2.6. Then $R_{k}$ will be smooth outside a finite subset, and we have $R_{k} \geq-\varepsilon_{k} \omega$ (resp. $R_{k} \geq \varepsilon \omega$ when $R$ is a Kähler current). Near each singular point of $R_{k}$, let us write $R_{k}=d d^{c} \varphi_{k}$ for some local potential $\varphi_{k}$. Then we replace $\varphi_{k}$ by $\max _{\eta}\left(\varphi_{k},-C_{k}\right)$, with $C_{k}>0$ big enough so that the gluing condition 
is satisfied, and the new $R_{k}$ thus obtained is smooth with $R_{k} \geq-\varepsilon_{k} \omega$. In the second case where $R_{k} \geq \varepsilon \omega$, we choose some local potential $\varphi_{k}$ near each singular point, and replace it by $\max _{\eta}\left(\varphi_{k},|z|^{2}-C_{k}\right)$ with $C_{k}>0$ big enough so that the gluing condition is satisfied, and we get in this way a smooth $R_{k}$ with a strictly positive lower bound, i.e. a Kähler form. The above reasoning shows in particular that $X$ is Fujiki iff it is Kähler.

Now if we assume that $\int_{X} T_{a c}^{2}>0$, then since $\{R\}$ is nef we get by Lemma $4.4\{R\}^{2} \geq \int_{X} R_{a c}^{2}=\int_{X} T_{a c}^{2}>0$, thus the intersection form on $H^{1,1}(X, \mathbf{R})$ cannot be negative definite. Now this compels $b_{1}(X)$ to be even by classical results of Kodaira (cf. e.g. [Lam99]), and this in turn forces $X$ to be Kähler by the main result of [Lam99] or [Buc99]. Therefore the above conjecture is true on a surface. In particular, whenever $v(a)>0$ for some pseudoeffective class $a$, we can choose $T \in a$ such that $\int_{X} T_{a c}^{2}=v(a)$, and then we get $v(a) \geq v(\{R\})=\{R\}^{2} \geq \int_{X} T_{a c}^{2}=v(a)$, thus $a$ and $\{R\}$ have same volume. Let us summarize all this in the

Proposition 4.16 Let $X$ be a compact complex surface. Then for each pseudoeffective class a on $X$, there exists a "Zariski decomposition" $a=d+r$ where $d$ is an infinite series of real effective divisors and $r$ is a nef class with $v(a)=r^{2}$. Furthermore, as soon as $v(a)>0$, $a$ is big and $r$ can be taken to be a Kähler class.

\subsubsection{Behaviour of the volume in deformations}

In this last part we prove the following

Proposition 4.17 The volume is upper-semicontinuous on Kähler deformations in the following sense: if $\mathcal{X} \rightarrow S$ is a proper submersive Kähler map and $a$ is a pseudoeffective $(1,1)$-class on the central fibre $X_{0}=: X$, then one has

$$
v(a) \geq \limsup _{b \rightarrow a} v(b)
$$

where $b$ is a pseudoeffective $(1,1)$-class on some fibre $X_{t}$.

Proof: Upon shrinking the base $S$, we may assume that the deformation is topologically trivial, and that there exist Kähler metrics $\omega_{t}$ on $X_{t}$ depending smoothly on $t$; we normalize them so that $\int_{X_{t}} \omega_{t}^{n}=1$. Let then $b_{k}$ be a sequence of cohomology classes in $H^{2}(X, \mathbf{C})$ converging to $a$, and such that $b_{k}$ is a pseudoeffective $(1,1)$-class on $X_{t_{k}}$ for some sequence $t_{k}$ going to 0 , and that $v\left(b_{k}\right)$ converges to the right hand side of our inequality. By the degenerate Calabi-Yau theorem, we can choose a closed positive $(1,1)$-current $T_{k} \in b_{k}$ on $X_{t_{k}}$ such that $T_{k, a c}^{n}=v\left(b_{k}\right) \omega_{t_{k}}^{n}$ almost everywhere. Since $b_{k}$ 
converges, it is bounded, thus $T_{k}$ is bounded in mass, and after extracting some subsequence, we may assume that $T_{k}$ converges to a closed positive $T \in a$. Then we apply Lemma 3.5 to get in the limit $T_{a c}^{n} \geq \lim \sup _{b \rightarrow a} v(b) \omega^{n}$, which gives the expected result.

As a consequence, we get the following "quantitative" version of a result of Huybrechts [Huy01]: denote by $\mathcal{C}_{X}$ the connected component of the Kähler cone in the open cone $\left\{a \in H^{1,1}(X, \mathbf{R}), \int_{X} a^{n}>0\right\}$. Then we have:

Proposition 4.18 If $X$ is an irreducible symplectic (Kähler) $2 n$-dimensional manifold, then $v(a) \geq \int_{X} a^{2 n}$ for each $a \in \mathcal{C}_{X}$.

Indeed, we can choose a sequence of very general points $t_{k}$ converging to 0 in the universal deformation space of $X$ such that $a$ is a limit of $(1,1)$ classes $a_{k}$ on $X_{t_{k}}$. By [Huy01], the cone $\mathcal{C}_{X_{t_{k}}}$ coincides with the Kähler cone of $X_{t_{k}}$ because $t_{k}$ is very general, therefore $a_{k}$ is a Kähler class and we have $v\left(a_{k}\right)=\int_{X} a_{k}^{2 n}$ for each $k$. The result then follows from Proposition 4.17.

I would like here to address my warmest thanks to my thesis advisor JeanPierre Demailly for his many suggestions and his interest in this work.

\section{References.}

- [Bon93] Bonavero, L. - Inégalités de Morse holomorphes singulières, C. R. Acad. Sci. Série I 317 (1993), 1163-1166.

- [Buc99] Buchdahl, N. - On compact Kähler surfaces, Ann. Inst. Fourier 50 (1999), 287-302.

- [Dem82] Demailly, J.-P. - Estimations $L^{2}$ pour l'opérateur $\bar{\partial}$ d'un fibré vectoriel holomorphe semi-positif au dessus d'une variété kählerienne complète, Ann. Sci. Ecole Norm. Sup. 15 (1982), 457-511.

- [Dem92] Demailly, J.-P. - Regularization of closed positive currents and intersection theory, J. Alg. Geom. 1 (1992), 361-409.

- [Dem93] Demailly, J.-P. - A numerical criterion for very ample line bundles, J. Diff. Geom. 37 (1992), 323-374.

- [Dem96] Demailly, J.-P. - in Introduction à la théorie de Hodge, Panoramas et synthèses, S.M.F. 3 (1996), 3-111.

- [DEL00] Demailly, J.-P.; Ein, L.; Lazarsfeld, R. - A subadditivity property of multiplier ideals, math.AG/0002035 (2000). 
- [DP01] Demailly, J.-P.; Paun, M. - Numerical characterization of the Kähler cone of a compact Kähler manifold, math.AG/0105176 (2001).

- [Fuj94] Fujita, T. - Approximating Zariski decomposition of big line bundles, Kodai Math. J. 17 (1994), 1-3.

- [Huy01] Huybrechts, D. - Erratum: Compact hyperkähler manifolds: basic results, math.AG/0106014 (2001).

- [JS93] Ji, S.; Shiffman, B. - Properties of compact complex manifolds carrying closed positive currents, J. Geom. Anal. 3, 1 (1993), 37-61.

- [Lam99] Lamari, A. - Courants kähleriens et surfaces compactes, Ann. Inst. Fourier 49 (1999), 249-263.

- [Mou98] Mourougane, C. - Versions kähleriennes du théorème d'annulation de Bogomolov, Collect. Math. 49, 2-3 (1998), 433-445.

- [Yau78] Yau, S.-T. - On the Ricci curvature of a complex Kähler manifold and the complex Monge-Ampère equation, Comm. Pure Appl. Math. 31 (1978). 